\title{
Incidence and risk predictors for osteonecrosis of the jaw in cancer patients treated with intravenous bisphosphonates
}

\author{
Marcin Kos
}

Department of Maxillofacial Surgery, Klinikum Minden, Minden, Germany

Submitted: 15 March 2013

Accepted: 23 April 2013

Arch Med Sci 2015; 11, 2: 319-324

DOI: 10.5114/aoms.2015.50964

Copyright $@ 2015$ Termedia \& Banach

\section{Abstract}

Introduction: The aim of this study was to establish the incidence of bisphosphonate-related osteonecrosis of the jaws (BRONJ) in oncologic patients and to determine risk predictors with respect to this condition.

Material and methods: This retrospective review included 197 oncologic patients treated from January 2005 to December 2010 with administration of bisphosphonates (BPs) as part of management. Sex, age, type of cancer diagnosed, period of substantial disease, oral surgery, type of bisphosphonate, number of doses, and cases of BRONJ diagnosis were recorded. The cumulative incidence and incidence rate of BRONJ were calculated. The factors that influenced BRONJ were assessed with multivariate logistic regression and with estimations of $95 \%$ confidence intervals and odd ratios. Values of $p \leq 0.05$ were considered significant.

Results: The BRONJ appeared in $9.64 \%$ of patients. The BRONJ incidence rate was 1 in 28 patients per year of BP treatment. Logistic regression showed that the odds of osteonecrosis increased 1.0172-fold with each given dose of BP. The BRONJ risk with zoledronate was 5 -fold higher than that with pamidronate or ibandronate. The risk of BRONJ increased by 40 -fold after dental surgery.

Conclusions: Period of BP administration and type of BP used are important risk predictors for the development of BRONJ in oncologic patients treated with intravenous administration of these drugs. Patient-related factors are dental or periodontal events connected with need for oral surgery.

Key words: zoledronate, bone, necrosis, risk.

\section{Introduction}

Bisphosphonates (BPs) originate from naturally occurring pyrophosphates by oxygen/carbon exchange in the basic pyrophosphate chain. It provides resistance against hydrolysis from bone phosphatases. Due to that the deposition of BPs in bone lasts several years. Bisphosphonates occur in different forms based on the type of side chain (R1 or R2) attached to the central carbon atom. The R1 side chain confers the affinity of BPs for calcium on bone surfaces; the complexity of the R2 structure confers its potency against bone resorption. Bisphosphonates with a primary amino group are up to two orders of magnitude more potent than those without an amino group. Methylation of the amino group or its inclusion within a heterocyclic ring further increases BP po-

\author{
Corresponding author: \\ Marcin Kos MD, PhD \\ Department \\ of Maxillofacial Surgery \\ Klinikum Minden \\ Hans-Nolte-Strasse 1 \\ 32429 Minden, Germany \\ Phone: + 4944140377668 \\ Fax: +49 4414032621 \\ E-mail: mkos@poczta.onet.pl
}


tency. Therefore, BPs that contain a secondary or tertiary amino group are among the most potent antiresorptive BPs [1].

Due to their unique ability to inhibit bone resorption, BPs have been used for several years in the treatment of bone metabolic disorders, primary or metastatic bone tumors and hypercalcemia of malignancy [2-4]. Bisphosphonates were originally considered safe; however, since 2003, patients who received BPs have increasingly exhibited large, therapy-resistant jaw bone exposures. This phenomenon, termed bisphosphonate-related osteonecrosis of the jaw (BRONJ), was first observed after infusions of potent, nitrogen-containing BPs [5]. However, the genesis, epidemiology, and factors that affect the course of this disease are not well characterized. There are few reports on the incidence rate of BRONJ, and the reported cumulative incidence has varied considerably in studies that differed in the type of disease being treated, the population assessed, and the methodology applied [6-10].

The objective of this study was to establish the cumulative incidence and incidence rate of BRONJ and to determine risk predictors with respect to this condition.

\section{Material and methods}

\section{Study design}

This was a retrospective review of 197 patients with cancer treated in the Department of Oncology and Hematology, Klinikum Minden, Germany, between 1 January 2005 and 31 December 2010. In these patients the dental status was monitored during oncologic therapy at the Department of Maxillofacial Surgery, Klinikum Minden, Germany.

\section{Inclusion criteria}

All cancer patients who had received intravenously at least 3 doses of BPs were included in this study. As part of the cancer treatment protocol, the patients received $60 \mathrm{mg}$ or $90 \mathrm{mg}$ of pamidronate intravenously every 4 weeks. Infusions of $4 \mathrm{mg}$ of zoledronate or $6 \mathrm{mg}$ of ibandronate were performed at the same intervals, so the number of doses was equal to months of BP administration. Of the 197 patients, 20 received more than one type of BP. Bisphosphonate-related osteonecrosis of the jaw was diagnosed when BP administration was followed by bone exposure that did not heal within 8 weeks after identification.

\section{Exclusion criteria}

Patients with a history of radiation therapy to the head and neck region and those who had received BPs for management of osteoporosis were excluded from the study.

\section{Data collection and calculations}

The following data were collected from medical records: sex, age, type of cancer diagnosis, period of substantial disease, oral surgery, type of BP, duration of $\mathrm{BP}$ administration in months (i.e., number of doses), and BRONJ diagnosis.

The cumulative incidence of BRONJ was calculated as the number of patients diagnosed with BRONJ divided by the total number of patients treated with BPs over the study period. The incidence rate of BRONJ was defined as the number of patients diagnosed with BRONJ divided by the total number of BP administrations in all patients.

\section{Statistical analysis}

Baseline differences between patients with and without BRONJ were assessed using the $\chi^{2}$ test for categorical variables and Mann-Whitney rank sum test for continuous variables. A $p$-value $\leq 0.05$ was considered significant.

Risk predictors of BRONJ were identified using multivariate logistic regression with stepwise backward reduction and with estimations of odds ratios (ORs) and 95\% confidence intervals $(\mathrm{Cl})$. Independent variables were chosen based on suspected risk factors; these included sex, age, oncological diagnosis, duration of the disease, time of treatment, and type of BPs given. Variables that were associated with BRONJ occurrence at $p \leq 0.1$ in univariate logistic regression were entered into the multivariate model. Variables at $p \leq 0.05$ were retained in the reduced model. SigmaStat software version 2.0 was used for the analysis.

The study was conducted in full accordance with ethical principles of the Declaration of Helsinki (version 2008).

\section{Results}

There were 197 patients with cancer (112 multiple myelomas, 62 breast carcinomas, 8 prostate carcinomas, 4 bronchial carcinomas, 4 colorectal carcinomas, 3 kidney clear cell carcinomas, 1 liver carcinoma, 1 urinary bladder carcinoma, 1 nonHodgkin's lymphoma, and 1 acute lymphoblastic leukemia). Of these, 19 patients developed BRONJ. The clinical characteristics of the study group are shown in Table I.

The cumulative incidence of BRONJ was 9.64\%. Among the different types of cancer, BRONJ occurred in $10.71 \%(12 / 112)$ of multiple myelomas, $9.68 \%(6 / 62)$ of breast carcinomas, and $6.67 \%$ $(1 / 15)$ of other neoplasms. The incidence rate of BRONJ in the evaluated population was 1 in 28 patients per year of BP treatment. Patients with BRONJ had experienced longer median durations of malignant disease ( $p=0.008$; Mann-Whitney rank sum test) and longer periods of BP adminis- 
Table I. Demographic and clinical characteristics among 197 patients receiving BP treatment as management of cancer

\begin{tabular}{|c|c|c|c|}
\hline Parameter & BRONJ $(n=19)$ & Non-BRONJ $(n=178)$ & Value of $p$ \\
\hline Sex [men : women] & $10: 9(52 \%: 48 \%)$ & $66: 112(37 \%: 63 \%)$ & 0.282 \\
\hline \multicolumn{4}{|l|}{ Diagnosis, $n(\%)$ : } \\
\hline Multiple myeloma & $12(63.1)$ & $100(56.2)$ & 0.917 \\
\hline Breast carcinoma & $6(31.6)$ & $56(31.5)$ & 0.811 \\
\hline Prostate carcinoma & $08(4.5)$ & 0.764 & \\
\hline Other tumors & $1(5.3)$ & $14(7.8)$ & 0.938 \\
\hline Dental events during BPs treatment ${ }^{1}, n(\%)$ & $15(79)$ & $6(3)$ & $\leq 0.001^{2}$ \\
\hline \multicolumn{4}{|l|}{ Age [years]: } \\
\hline Mean & 67.39 & 65.8 & 0.925 \\
\hline Range & $48-77$ & $41-92$ & \\
\hline \multicolumn{4}{|l|}{ Duration of the oncologic disease [months]: } \\
\hline Mean & 93.16 & 53.48 & 0.008 \\
\hline Range & $16-252$ & $3-360$ & \\
\hline \multicolumn{4}{|l|}{ Period of BP administration [months]: } \\
\hline Mean & 34.25 & 28.2 & 0.001 \\
\hline Range & $12-89$ & $3-121$ & \\
\hline
\end{tabular}

${ }^{1}$ dentified dental events were: teeth extractions (11 pts), implant insertion (1 pt), dental abscess (1 pt), ill fitting denture (1 pt), tooth-root apicotomy (1 pt). ${ }^{2}$ Statistical significance is printed in boldface.

tration, i.e., a higher number of given doses ( $p=$ 0.001; Mann-Whitney rank sum test). No BRONJ occurred in patients taking BPs for less than 12 given doses.

In $78.9 \%$ of cases ( $n=15)$, BRONJ development was preceded by dental events that required oral surgical procedures (Figure 1); but in 6 similar cases, BRONJ did not develop, despite the fact that BP therapy had continued.

The multivariate logistic regression model showed that patient age, type of BP, number of given doses, and oral surgery during BP treatment were important risk predictors for BRONJ (Table II). The odds for osteonecrosis were estimated to increase by 1.0427 -fold with each year of age and by 1.0172-fold with each dose of BP. The risk of BRONJ with zoledronate was 5-fold higher than that of pamidronate or ibandronate. Any kind of dental surgery performed during treatment with BPs was connected with an average 40-fold increase in the risk of BRONJ development.

\section{Discussion}

Cooperation between the Department of Hematology and Oncology and the Department of Maxillofacial Surgery was established to maintain the oral health of oncologic patients before and during chemotherapy. The present analysis used

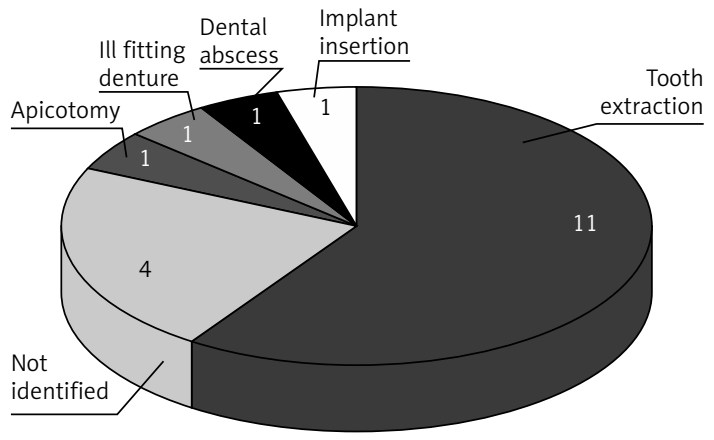

Figure 1. Distribution of the preceding dental events which were identified in $15(78.9 \%)$ of all $(n=19)$ patients who developed BRONJ

the data recorded during the treatment to evaluate the hazard, frequency, and risk predictors for BRONJ. The main strength of this study was that the data collection was achieved from a well-defined population of patients with cancers who had been treated in one center with intravenous BP therapy, which considerably lowered the possibility of collection of misleading data. A limitation of the study resulting from such an approach was that a restricted number of patients could be included. A larger study might have identified differences that did not reach statistical significance in this population. 
Table II. Multivariate model exploring risk predictors for BRONJ among patients with cancer treated with BPs $(n=197)$

\begin{tabular}{|lccc|}
\hline Predictor & Odds ratio & $95 \% \mathrm{Cl}$ & Value of $p$ \\
\hline Sex & 1.0909 & $0.5229 ; 2.2761$ & 0.81 \\
\hline Age & 1.0427 & $1.0058 ; 1.0809$ & $0.023^{1}$ \\
\hline Oncologic diagnosis & 1.3735 & $0.9674 ; 1.9501$ & 0.08 \\
\hline Duration of the oncologic disease & 1.0032 & $0.9979 ; 1.0086$ & 0.242 \\
\hline $\begin{array}{l}\text { Treatment with zoledronate } \\
\text { vs. pamidronate }\end{array}$ & 4.5951 & $1.5939 ; 7.2250$ & $<0.001$ \\
\hline Number of given doses & 1.0172 & $1.0018 ; 1.0328$ & 0.023 \\
\hline Oral surgery & 44.433 & $17.366 ; 113.68$ & $<0.001$ \\
\hline
\end{tabular}

${ }^{1}$ Statistical significance is printed in boldface.

The cumulative incidence was $9.64 \%$ in the studied population. The cumulative incidence reported in the literature varied considerably. It appeared to be lower in retrospective or web-based studies than in prospective trials and to be highest in patients who received BPs for multiple myeloma, breast carcinoma, or prostate carcinoma $[6,7,9,11]$. Conversely, patients treated for benign bone conditions showed relatively low BRONJ incidence. This may have been due to the lack of oncologic treatment or because a relatively low cumulative dose of BP was sufficient for a therapeutic effect $[3,12,13]$.

This study showed that older age was associated with the risk of BRONJ. This could be because regenerative and healing processes decrease with age. Furthermore, in older patients, complications of dental caries and periodontal diseases can increase the need for dental surgery and the use of dentures; both these are recognized risk factors for BRONJ [14-16]. However, older age of the patients presenting BRONJ reflected a longer disease course and consequently a longer exposure time to BPs. From this point of view it could not be regarded as a risk predictor in its own right. Alternatively, larger studies are needed to support the role of age in the development of BRONJ [17].

Bisphosphonates potency and number of given doses had an impact on the development of BRONJ. The multivariate analysis presented here showed a 5-fold increase in the risk of BRONJ development after administration of a more potent zoledronate compared with pamidronate and ibandronate. The other studies showed that patients who had ever received zoledronate had a 4.5-28-fold increase in relative risk for BRONJ development $[7,8,10,11]$. However, recent reports on denosumab, an antibody that potently inhibits osteoclast differentiation and function, showed that the risk of developing osteonecrosis was more likely dependent on the degree of osteoclast-mediated inhibition of bone turnover than on the type of inhibitor. They observed similar rates of bone necrosis with zoledronate and denosumab treatments [18].

In the present study, patients with BRONJ had longer (34 months) BP treatments (i.e., a higher number of doses) than those without BRONJ (28 months). Other studies confirmed that longer periods of BP treatment were associated with higher risk of BRONJ development. In the study by Bamias et al. [7], the median duration of BP therapy before the occurrence of osteonecrosis was 35 months, and the median BP therapy duration was 15 months in patients who did not develop BRONJ; and no patients who received less than 13 infusions developed bone complications. Those results were consistent with the results of the present study, where no case of osteonecrosis was found in patients treated for less than 12 months (i.e., 12 doses). Such a finding was confirmed in the study of Then et al. [17]. According to Vahtsevanos et al., development of BRONJ was unlikely with less than 6 months of BP treatment [10].

The course of BRONJ gives rise to the question of whether exposure to BPs is sufficient to develop BRONJ. Although the disease can occur spontaneously, it is mostly provoked (in this study in $78 \%$ of cases) by any kind of dental event [7, 8, 10, $17,19,20]$. Most patients with BRONJ had experienced recent tooth extractions, root-tip resections, or cystectomies with subsequent wound healing problems and presentation of wound dehiscence with bone exposure. Clinical symptoms may also be preceded by local trauma, or pressure from ill fitting dentures. This study showed that the presence of any of the above factors followed by dental surgery caused an average 40-fold increase in the risk of BRONJ development. There is growing evidence that infections might play a role in the pathogenesis of BRONJ [17, 20], but it is likely that the disease may not have developed in the absence of an initial bone exposure. Recently, it was proved that administration of BPs causes 
increased bacterial adhesion to hydroxyapatite coated with these agents [21]. It promotes bacterial colonization of the denuded bony surface, thus creating favorable conditions for the development of chronic and therapy resistant infection. Bone exposure during the surgery acts as a trigger opening the door for bacterial invasion. This could explain the reported strong correlation between BRONJ and dental surgical procedures. In the light of the above facts, jaw bones are especially subjected to infection when compared with other parts of the skeleton, because they easily come into direct contact with the external environment due to the thin epithelial lining coating their surface, susceptibility to trauma, and presence of teeth $[16,17,19$, 21-23]. Therefore, it is uncertain whether BPs were solely responsible for the bone effects, particularly because the patients were simultaneously experiencing other potentially osteonecrotic or inflammatory co-morbidities due to oncologic disease, chemotherapy, administration of corticosteroids or stem cell transplantation [17, 24-26]. Other nonspecific risks may include vascular disorders, diabetes, smoking, or malnutrition [27].

In conclusion, the present results showed that the period of BP administration and type of bisphosphonate used were important risk predictors for the development of BRONJ in oncologic patients treated with intravenous administration of these drugs. Patient-related factors are dental or periodontal events connected with dental surgical procedures [16].

\section{Acknowledgments}

The author is grateful to Prof. Dr. M. Griesshammer, Head of the Department of Hematology and Oncology, and PD Dr. W. Engelke, Head of the Department of Maxillofacial Surgery, Klinikum Minden, for their substantial support and establishment of cooperation between both units, which was essential for this study.

\section{Conflict of interest}

The author declares no conflict of interest.

\section{References}

1. Graham R, Russell G. Bisphosphonates: mode of action and pharmacology. Pediatrics 2007; 119: 150-62.

2. Baczyk G, Opala T, Kleka P, Chuchracki M. Multifactorial analysis of risk factors for reduced bone mineral density among postmenopausal women. Arch Med Sci 2012; 8: 332-41.

3. Kos M, Luczak K, Godzinski J, Klempous J. Treatment of monostotic fibrous dysplasia with pamidronate. J Craniomaxillofac Surg 2004; 32: 10-15.

4. Wu S, Dahut WL, Gulley JL. The use of bisphosphonates in cancer patients. Acta Oncol 2007; 46: 581-91.
5. Marx RE. Pamidronate (Aredia) and zoledronate (Zometa) induced avascular necrosis of the jaws: a growing epidemic. J Oral Maxillofac Surg 2003; 61: 1115-7.

6. Durie BG, Katz M, Crowley J. Osteonecrosis of the jaw and bisphosphonates. N Engl J Med 2005; 353: 99-102.

7. Bamias A, Kastritis E, Bamia C, et al. Osteonecrosis of the jaw in cancer after treatment with bisphosphonates: incidence and risk factors. J Clin Oncol 2005; 23: 8580-7.

8. Zervas K, Verrou E, Teleioudis Z, et al. Incidence, risk factors and management of osteonecrosis of the jaw in patients with multiple myeloma: a single-centre experience in 303 patients. Br J Haematol 2006; 134: 20-3.

9. Wang EP, Kaban LB, Strewler GJ, Raje N, Troulis MJ. Incidence of osteonecrosis of the jaw in patients with multiple myeloma and breast or prostate cancer on intravenous bisphosphonate therapy. J Oral Maxillofac Surg 2007; 65: 1328-31.

10. Vahtsevanos K, Kyrgidis A, Verrou E, et al. Longitudinal cohort study of risk factors in cancer patients of bisphosphonate-related osteonecrosis of the jaw. J Clin Oncol 2009; 27: 5356-62.

11. Hoff AO, Toth B, Hu M, Hortobagyi GN, Gagel RF. Epidemiology and risk factors for osteonecrosis of the jaw in cancer patients. Ann N Y Acad Sci 2011; 1218: 47-54.

12. Schwartz S, Joseph C, lera D, Vu DD. Bisphosphonates, osteonecrosis, osteogenesis imperfecta and dental extractions: a case series. J Can Dent Assoc 2008; 74: 537-42.

13. Malmgren B, Astrom E, Soderhall S. No osteonecrosis in jaws of young patients with osteogenesis imperfecta treated with bisphosphonates. J Oral Pathol Med 2008; 37: $196-200$.

14. Elad S, Thierer T, Bitan M, Shapira MY, Meyerowitz C. A decision analysis: the dental management of patients prior to hematology cytotoxic therapy or hematopoietic stem cell transplantation. Oral Oncol 2008; 44: 37-42.

15. Aghaloo TL, Kang B, Sung EC, et al. Periodontal disease and bisphosphonates induce osteonecrosis of the jaws in the rat. J Bone Miner Res 2011; 26: 1871-82.

16. Kos M. Association of dental and periodontal status with bisphosphonate related osteonecrosis of the jaws. A retrospective case controlled study. Arch Med Sci 2014; 10: 117-23.

17. Then C, Hörauf N, Otto S, et al. Incidence and risk factors of bisphosphonate-related osteonecrosis of the jaw in multiple myeloma patients hawing undergone autologous stem cell transplantation. Onkologie 2012; 35: 658-64.

18. Stopeck AT, Lipton A, Body JJ, et al. Denosumab compared with zoledronic acid for the treatment of bone metastases in patients with advanced breast cancer: a randomized, double-blind study. J Clin Oncol 2010; 28 : 5132-9.

19. Kos M, Kuebler JF, Luczak K, et al. Bisphosphonate-related osteonecrosis of the jaws: a review of 34 cases and evaluation of risk. J Craniomaxillofac Surg 2010; 38: 255-9.

20. Hoff AO, Toth BB, Altundag K, et al. Frequency and risk factors associated with osteonecrosis of the jaw in cancer patients treated with intravenous bisphosphonates. J Bone Miner Res 2008; 23: 826-36.

21. Kos M, Junka A, Smutnicka D, Bartoszewicz M, Kurzynowski T, Gluza K. Pamidronate enhances bacterial adhesion to bone hydroxyapatite. Another puzzle in the pathology of bisphosphonate-related osteonecrosis of the jaw? J Oral Maxillofac Surg 2013; 71: 1010-6. 
22. Kos M. Bisphosphonates promote jaw osteonecrosis through facilitating bacterial colonisation. Med Hypotheses 2011; 77: 214-5.

23. Kos M, Brusco D, Kuebler JF, Engelke W. Clinical comparison of patients with osteonecrosis of the jaws, with and without a history of bisphosphonates administration. Int J Oral Maxillofac Surg 2010; 39: 1097-102.

24. Engel IA, Straus DJ, Lacher M, Lane J, Smith J. Osteonecrosis in patients with malignant lymphoma: a review of twenty-five cases. Cancer 1981; 48: 1245-50.

25. Mirzai R, Chang C, Greenspan A, Gershwin ME. The pathogenesis of osteonecrosis and the relationships to corticosteroids. J Asthma 1999; 36: 77-95.

26. Tauchmanova L, de Rosa G, Serio B. Avascular necrosis in long-term survivors after allogeneic or autologous stem cell transplantation: a single center experience and a review. Cancer 2003; 97: 2453-61.

27. Khamaisi M, Regev E, Yarom N, et al. Possible association between diabetes and bisphosphonate-related jaw osteonecrosis. J Clin Endocrinol Metab 2007; 92: 1172-5. 